\title{
Téoros
}

Revue de recherche en tourisme

\section{L'attractivité touristique des territoires}

\section{Serge Gagnon}

Volume 26, numéro 2, été 2007

L'attractivité touristique des territoires

URI : https://id.erudit.org/iderudit/1070939ar

DOI : https://doi.org/10.7202/1070939ar

Aller au sommaire du numéro

Éditeur(s)

Université du Québec à Montréal

ISSN

0712-8657 (imprimé)

1923-2705 (numérique)

Découvrir la revue

Citer ce document

Gagnon, S. (2007). L'attractivité touristique des territoires. Téoros, 26(2), 3-4. https://doi.org/10.7202/1070939ar d'utilisation que vous pouvez consulter en ligne.

https://apropos.erudit.org/fr/usagers/politique-dutilisation/ 


\section{L'attractivité touristique des territoires}

\section{Serge Gagnon}

Peu d'études ont été publiées sur la question de l'attractivité touristique des territoires, tout au moins en France et au Québec. On constate généralement que les acteurs du tourisme fondent leurs actions sur un certain nombre de croyances, en particulier sur ce qui concerne l'attractivité des destinations touristiques. On trouve encore régulièrement par exemple la référence à une vision strictement «émettrice » de l'image des destinations, sans prendre en compte la nécessité d'évaluer les composantes de cette image dans l'esprit des destinataires de messages: l'image "projetée" prend le pas sur l'image "émergente", sans que cette dernière soit, la plupart du temps, analysée précisément.

Il est généralement admis aussi que l'attraction touristique d'un territoire renvoie à une diversité d'équipements, d'acteurs, d'actions, d'événements qui provoquent des déplacements. Comment le corpus assume-t-il cette diversité phénoménale? En explicitant, en chaque situation donnée, la spécificité des déplacements. En effet, les phénomènes touristiques sont généralement rapportés à une multiplicité de trajets entre lieux de départ et d'arrivée, soit ce qu'évoque le couplage "origine-destination ». Soumise à des modalités d'interaction ultimement économiques, l'attraction touristique d'un territoire doit-elle se limiter uniquement au traitement empirique des faits? Certainement non. L'attraction touristique d'un territoire est une réalité complexe. Mais comment aller de la diversité phénoménale des faits et des événements touristiques à une connaissance qui puisse prendre en charge la compréhension de cette attractivité ?

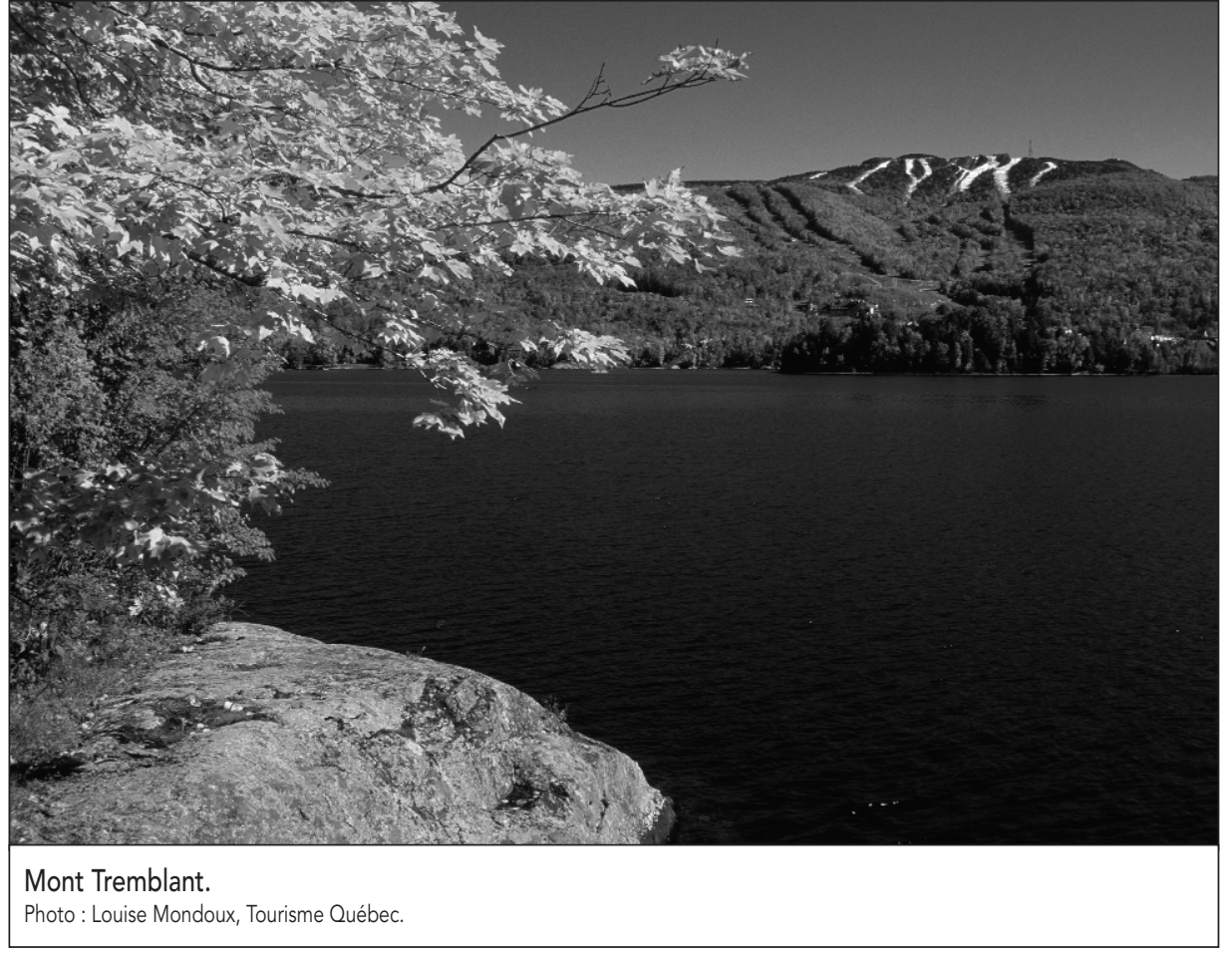

Vous est-il arrivé de visiter fréquemment des régions telles que Charlevoix, les Laurentides ou le Saguenay? Vous êtesvous déjà demandé pourquoi certains territoires attirent plus que d'autres? L'attractivité touristique d'un territoire dépend certes des qualités naturelles et spectaculaires d'un site, comme le majestueux fjord du Saguenay ou les chutes du Niagara, en Ontario. Cependant, l'agrément d'un territoire réputé pour ses charmes doit davantage à une ambiance qui motive le touriste à se conjoindre à ces lieux. Comment alors peut-on qualifier la dimension attractive d'un territoire?

De nouveaux regards convergent, ils envisagent la nécessité de rompre avec les études qui approchent la question de l'attractivité sous un aspect exclusivement discipli- naire, en vase clos. Les chercheurs s'entendent qu'il faut une approche plus globale, multidisciplinaire, pour aborder cette problématique centrale en tourisme, ce que ce numéro de la revue Téoros devrait permettre. L'objectif de ce numéro consiste donc à décloisonner le regard porté sur l'attractivité touristique des territoires, c'est-à-dire de transcender les descriptions et les explications sectorielles, lesquelles n'ont pas réussi à constituer une assise solide pour l'interprétation de cette problématique. Pour comprendre davantage les processus fondamentaux qui influencent l'attrait d'un territoire pour le tourisme, le présent numéro propose d'en explorer quelques conditions de possibilité: anthropologique, historique, géographique, économique, politique et sociologique. 
Pour ce faire, le numéro comprend trois parties. La première propose une exploration de la piste théorique du problème de l'attractivité. Serge Gagnon de l'Université du Québec en Outaouais, nous fait faire un voyage aux fondements géo-anthropologiques de l'attractivité touristique des territoires, tandis que François de Grandpré de I'Université du Québec à Trois-Rivières établit des distinctions conceptuelles fondamentales pour orienter le développement touristique régional. La deuxième partie propose quatre études de cas européens. Vincent Calay de l'Université Libre de Bruxelles nous offre une dissertation sur les politiques de valorisation des trois "Capitales de l'Europe», tandis qu'Alain
Escadafal de l'Université Michel de Montaigne à Bordeaux explique les stratégies d'organisation territoriale de la France en relation avec l'attractivité des destinations touristiques. Pour finir cette partie, Erwan Charles et Hervé Thouément de l'Université de Bretagne occidentale à Brest présentent les résultats d'une étude sur les déterminants du label territorial de la Bretagne et Jean-Pierre Augustin de l'Université Bordeaux III dresse le portrait attractif d'une destination océane de la côte Aquitaine. La troisième et dernière partie se concentre sur deux études de cas québécois. Nathalie Lahaye de I'Université Paul-Sabatier, campus de Tarbes, explique, avec le cas du mont Orford, en quoi l'attractivité d'un terri- toire peut provoquer des conflits d'usages. Enfin, Fabienne Joliet de I'Institut National d'Horticulture d'Anger et Thibault Martin de I'Université du Québec en Outaouais font le point sur la relation entre les représentations du paysage et l'attractivité touristique, à partir du cas du mont Tremblant.

J'espère que ce numéro thématique ouvrira le débat sur une redéfinition du caractère territorial des attractions touristiques. Bonne lecture!

Serge Gagnon est professeur au département de travail social et des sciences sociales de I'Université de Québec en Outaouais.

\section{TÉQROS

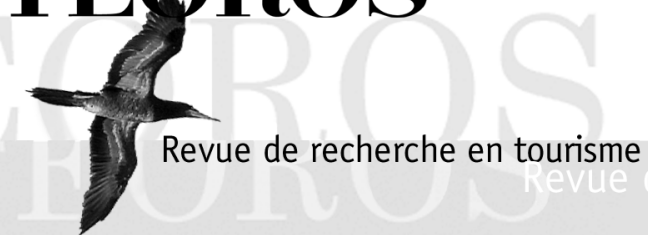

En dehors des dossiers thématiques TÉOROS ouvre ses pages aux propositions spontanées. La rédaction invite tous les chercheurs qui oeuvrent dans le domaine du tourisme ou qui s'intéressent au tourisme sous tous ses aspects, à soumettre des articles de nature analytique à la revue. On peut soumettre un article en l'envoyant à :

\section{teoros@uqam.ca}

Publiée trois fois l'an depuis 1982, la revue Téoros de recherche en tourisme s'affirme dans la francophonie comme le leader de diffusion des résultats de recherche et de réflexions fondamentales et appliquées dans une perspective multidisciplinaire. Les problématiques liées aux rapports entre le tourisme et la culture ou entre le tourisme et la société, à la gestion ou à la planification en tourisme, à des cas particuliers de mise en tourisme ainsi qu'aux questions méthodologiques d'actualité, par exemple, comptent parmi les sujets abordés.

Les textes soumis doivent apporter une contribution scientifique originale, que ce soit par le biais d'information factuelle, jusqu'alors inconnue ou par une nouvelle interprétation d'un thème particulier. Téoros vise avant tout le transfert de connaissances; son objectif est donc de promouvoir une meilleure compréhension des phénomènes liés au tourisme.

Les auteurs doivent faire parvenir un manuscrit présenté selon les règles de la revue, disponibles au www.teoros.uqam.ca.
Habituellement, un article analytique compte environ 6000 mots et n'excède pas 8000 mots avec trois ou quatre illustrations. On pourra cependant considérer des textes plus longs ou plus courts. Les articles peuvent être soumis en anglais ou en français et doivent être accompagnés d'un résumé de 100 à 200 mots (français/anglais) et de cinq mots-clés.

La publication des articles se fait sous réserve d'une évaluation. Tous les manuscrits seront évalués anonymement par des pairs qui pourront faire des suggestions ou demander des modifications. La rédaction transmettra l'avis des évaluateurs aux auteurs et s'assurera que les modifications demandées seront apportées.

Au plaisir de vous lire dans nos pages. 